\title{
A comparison of DNA and RNA quadruplex structures and stabilities
}

\author{
Astrid Joachimi, Armin Benz, Jörg S. Hartig* \\ Department of Chemistry and Konstanz Research School Chemical Biology (KoRS-CB), University of Konstanz, Universitätsstr. 10, 78457 Konstanz, Germany
}

Keywords:

DNA quadruplex

RNA quadruplex

Nucleic acids structure

\begin{abstract}
A B S T R A C T
Guanosine-rich sequences are prone to fold into four-stranded nucleic acid structures. Such quadruplex sequences have long been suspected to play important roles in regulatory processes within cells. Although DNA quadruplexes have been studied in great detail, four-stranded structures made up from RNA have received only minor attention, although it is known that RNA is able to form stable quadruplexes as well.

Here, we compare quadruplex structures and stabilities of a variety of DNA and RNA sequences. We focus on well established DNA sequences and determine the topologies and stabilities of the corresponding RNA sequences by $C D$ spectroscopy and $C D$ thermal melting experiments. We find that the RNA sequences exclusively fold into the all-parallel conformation in contrast to the diverse topologies adopted by DNA quadruplexes. The thermal stabilities of the RNA structures rival those of the corresponding DNA sequences, often displaying enhanced stabilities compared to their DNA counterparts. Especially thermodynamically less stable sequences show a strong preference for potassium, with the RNA quadruplexes exhibiting much higher stabilities than the corresponding DNAs. The latter finding suggests that quadruplexes formed at critical positions in mRNAs might perturb gene expression to a larger extend than previously anticipated.
\end{abstract}

\section{Introduction}

Guanine-rich sequences are prone to fold into four-stranded structures called quadruplexes or G-quartets. ${ }^{1.2}$ DNA quadruplexes can adopt manifold topologies and conformations, characterized by their relative strand orientation. For example, all-parallel, allantiparallel, as well as mixed conformations have been reported for the intramolecular quadruplex formed by the telomeric repeat, see Figure $1 .^{3-7} \mathrm{~A}$ regulatory function of quadruplex structures in biological systems has long been proposed for these telomeric repeats at the ends of the chromosomes. In addition, putative Gquadruplexes are found within several promoter regions of eukaryotic genomes. ${ }^{8-10}$ Interestingly, those sequences seem to be enriched in proto-oncogenes and on the other hand being more rarely found in tumor-suppressor genes. The most studied promoter-based quadruplex is located in the proto-oncogene c-myc, forming a mixed conformation consisting of both parallel and anti-parallel strand orientations. ${ }^{12.11,12}$ Furthermore, it has been demonstrated that the addition of quadruplex-stabilizing compounds reduces the level of $c$-myc transcription..$^{3-15}$

In addition to the investigation of naturally occurring DNA quadruplexes, systematic studies have provided a profound understanding of the features determining the thermodynamic stability

\footnotetext{
Corresponding author. Tel.: +4907531884574 .

E-mail address: joerg.hartig@uni-konstanz.de (J.S. Hartig).
}

of these four-stranded structures. For example, shorter loops increase its stability. ${ }^{16.17}$ Although DNA quadruplexes have been studied in great detail over the past 15 years, only little is known about the stability and folding of corresponding G-rich RNA sequences. Despite the common knowledge that G-rich RNA is able to adopt stable, four-stranded structures as well. ${ }^{18-21}$ no comparative study has been published that systematically compares DNA and RNA quadruplex stabilities. Due to the absence of the complementary strand as in genomic DNA competing by duplex formation with the intra-strand folding, the likelihood of RNA quadruplex formation in cellular RNAs should be increased. Some recent studies highlight the importance of RNA quadruplexes in cellular processes. Quadruplexes forming in mRNAs have, been found to interfere with gene expression in both pro- and eukaryotes, ${ }^{18.19,22-24}$ for a more detailed discussion see below. Recently, bioinformatics studies have provided evidence that putative RNA quadruplexes are more frequently found at certain positions in untranslated regions of mRNAs. ${ }^{25}$ In addition to mRNA-based quadruplexes, telomeres have been found to be actively transcribed by polymerase II, generating RNAs with the potential to form quadruplexes also involved in regulatory processes at the telomeres. ${ }^{26,27}$ Structures of the telomeric RNA repeat have been reported recently, displaying an all-parallel propeller conformation of the quadruplex. ${ }^{28,29}$ The observed conformation seems to be the most stable topology for all RNA quadruplexes since the ribose pucker favors the anti-conformation of the glycosidic bond, predominating in the all-parallel conformation. $^{28-30}$ 
A

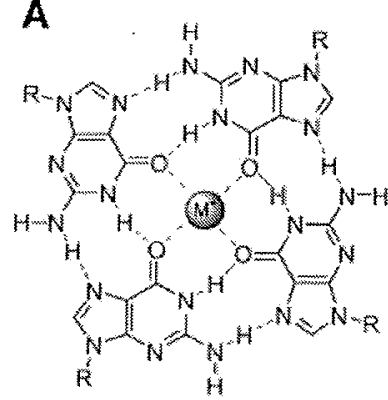

B

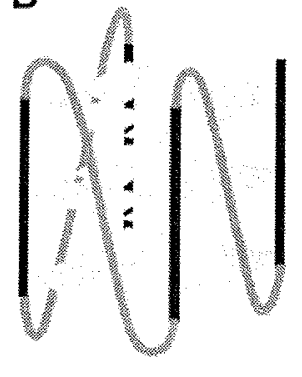

C

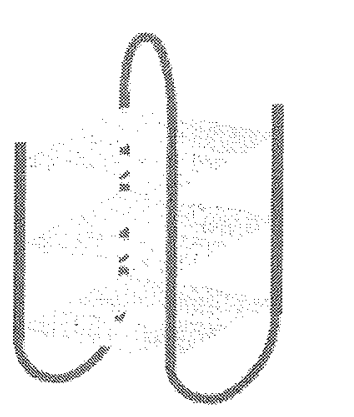

D

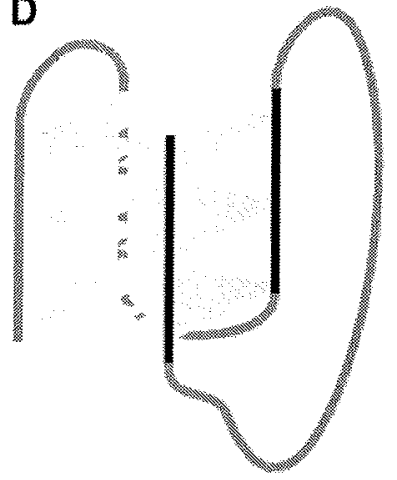

Figure 1. Schematic representation of quadruplex tetrad and topologies. (A) Tetrad composed of four guanine nucleobases and a monovalent ion coordinated by the 6 oxo-groups. (B) All-parallel strand orientation, also called propeller form. (C) Example of an all- anti-parallel quadruplex, the example resembles the so-called basket conformation. (D) Example of a mixed parallel-and anti-parallel quadruplex sometimes referred to as dogear conformation. Parallel-oriented strands are highlighted in black, schematic guanine tetrads are shown in light grey.

We are interested in the stability of such RNA quadruplexes since we are using quadruplex sequences as regulatory modules in order to control RNA functions. For example, we have engineered a hammerhead ribozyme that is under control of an RNA G-quadruplex-ligand interaction. ${ }^{31}$ In addition, we have used RNA quadruplexes for the in cis modulation of gene expression in Escherichia coli. ${ }^{18.19}$ In a further study, we used quadruplexes as small-molecule-addressable sequences for the regulation of dicer-mediated processing of G-rich shRNAs. ${ }^{32}$ In order to better judge the stabilities of RNA quadruplexes, we have carried out a comparative study by investigating DNA and RNA quadruplex sequences in the absence and presence of the monovalent ions potassium and sodium which are known to stabilize quadruplexes by coordination with the 6-oxo-functionality of the tetrad-forming guanosines.

\section{Results}

We have characterized model DNA quadruplex-forming sequences in comparison to their RNA counterparts. We focussed on sequences that are well characterized and fold into intramolecular quadruplexes since these are more interesting with respect to our applications as regulatory modules. As model sequences for our comparative study we have chosen nucleic acid sequences that are known to fold into well-characterized DNA quadruplex structures but vary in terms of thermodynamic stability. We decided to investigate the human $\mathrm{G}$-rich telomeric repeat, the most intensively studied G-rich sequence (termed HT, see Table 1). The DNA sequence folds into at least five different topologies that sometimes exist as mixtures depending on the buffer conditions and

Table 1

Sequences used in this study

\begin{tabular}{ll} 
Name & Sequence \\
\hline HT-DNA & GGGTTAGGGTTAGGGTTAGGG \\
HT-RNA & GGGUUAGGGUUAGGGUUAGGG \\
OXY-DNA & GGGGTTTGGGGTTTGGGGTTTTGGGG \\
OXY-RNA & GGGGUUUUGGGGUUUUGGGGUUUUGGGG \\
GQP1-DNA & GGGTGGGTGGGTGGG \\
GQP1-RNA & GGGUGGGUGGGUGGG \\
GOP2-DNA & GGTGGTGGTGG \\
GOP2-RNA & GGUGGUGGUGG \\
TBA-DNA & GGTTGGTGTGGTTGG \\
TBA-RNA & GGUUGGUGUGGUUGG \\
SDQ-DNA & GTGTGGGAAGGAGGGTGTGCATGGG \\
SDQ-RNA & GUGUGGGAAGGAGGGUGUGCAUGGG
\end{tabular}

a oligonucleotide sequences are shown in $5^{\prime}-3^{\prime}$-direction.

the exact sequence. ${ }^{1-7,33}$ Recently, it has been demonstrated that telomeric DNA gets transcribed and it is suspected that the resulting GGGUUA-repetitive RNAs are involved in regulatory functions at the telomeres as well. ${ }^{26,27}$ Two recent reports demonstrate that the corresponding RNA quadruplex composed of the four-repeat sequence adopts an all-parallel quadruplex. ${ }^{28.29}$ As second sequence we chose the Oxytricha telomeric repeat (termed OXY), a sequence that is known to form a quadruplex composed of four stacked G-tetrads comprising TTTT tetranucleotide loops in the solution as well as crystal structure. ${ }^{34,35}$ Third, we investigated a DNA G-quadruplex-forming aptamer specific for human $\alpha$-thrombin $^{36}$ (termed TBA) that is known to fold into a defined all-antiparallel conformation, the so-called chair topology. ${ }^{37}$ In addition to these sequences where several $X$-ray and solution structures are available for the respective DNA structures, we chose two model quadruplexes containing single-nucleotide T-loops being able to fold into very stable, all-parallel, intramolecular DNA quadruplexes of the propeller-type, ${ }^{17}$ termed GQP1 (containing three G-tetrads) and GQP2 (containing two G-tetrads, see Table 1). A further sequence we investigated is an example of an RNA quadruplex that we previously identified to control bacterial translation via masking of the Shine-Dalgarno sequence (termed SDQ for ShineDalgarno quadruplex). The RNA sequence has been shown to display intermediate thermal stability, being able to respond to temperature changes and thereby acting as an artificial RNA thermometer. ${ }^{18,19}$

The structures and stabilities of quadruplexes derived from the vertebrate telomeric repeat are well studied in case of the respective DNA sequences. The four-repeat sequence studied here shows a pronounced positive signal at $290 \mathrm{~nm}$ and a negative peak at $265 \mathrm{~nm}$ in the $\mathrm{CD}$ spectrum in $\mathrm{Na}^{+}$-containing buffer, indicative of an anti-parallel fold of the basket-type, see Figure $2 .{ }^{4}$ The $\mathrm{K}^{+}$spectrum shows a mixture of anti-parallel and parallel folds which has been observed frequently. ${ }^{1.27}$ In the absence of monovalent ions no significant folding is observed. In contrast to the DNA sequence, the corresponding RNA folds exclusively into the parallel topology irrespective of the type of monovalent ions, see Figure 2. The finding corresponds to the latest study describing the propeller structure of the telomeric RNA sequence for both potassium $^{29}$ as well as sodium-containing solutions. ${ }^{28}$ The stability of the RNA quadruplex is higher in case of $\mathrm{K}^{+}$and lower in case of $\mathrm{Na}^{+}$-containing solutions compared to the DNA counterpart. In contrast to the vertebrate sequence, the quadruplex formed by the Oxytricha telomeres exclusively folds into an anti-parallel conformation irrespective of the type of monovalent ions, see Figure 2. The results are in accordance with a basket-type conformation for the intramolecular quadruplex found in high resolution NMR studies. ${ }^{38.34}$ The RNA sequence again adopts only the all-parallel topology. The stabilities of the DNA sequence are slightly higher 
A
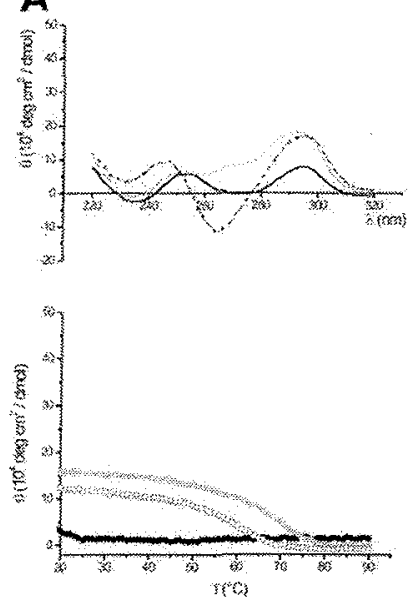

B
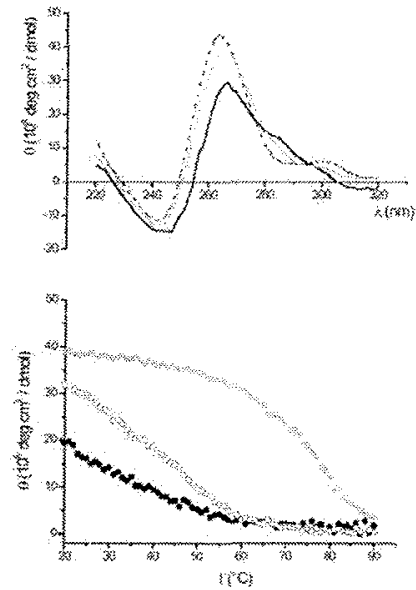

C
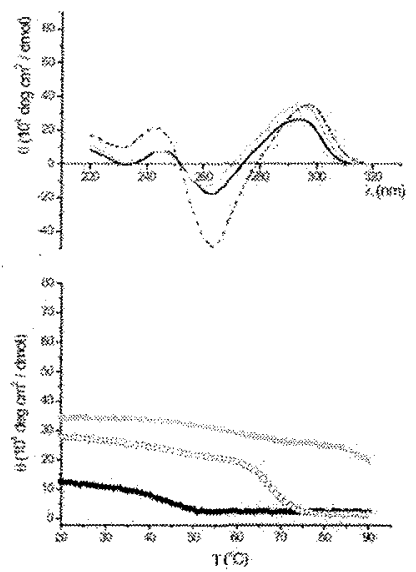

D
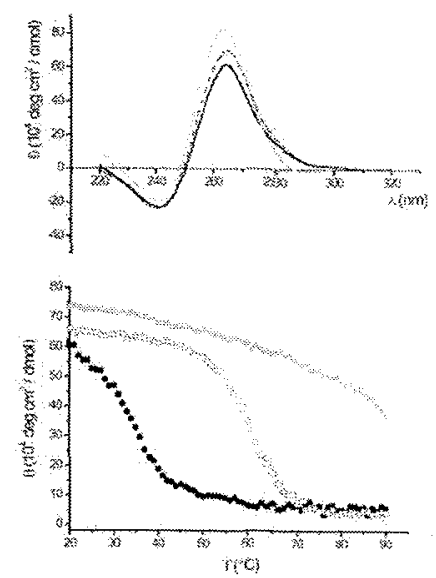

Figure 2. Vertebrate and Oxytricha telomeric sequences. (A) Vertebrate telomeric DNA (HT DNA). (B) Vertebrate telomeric RNA (HT RNA). (C) Oxytricha telomeric DNA (OXY DNA). (D) Oxytricha telomeric RNA (OXY RNA). Upper panels: CD spectra without monovalent ions (black line), containing $100 \mathrm{mM} \mathrm{NaCl}$ (dashed line) and $100 \mathrm{mM} \mathrm{KCl}$ (grey line). Lower panels: $\mathrm{CD}$ melting experiments carried out at the wavelength of the most pronounced peak in the upper CD spectra: without monovalent ions (black circles), containing $100 \mathrm{mM} \mathrm{NaCl}$ (open circles) and $100 \mathrm{mM} \mathrm{KCl}$ (grey circles).

compared to the RNA quadruplexes (approx. $5^{\circ} \mathrm{C}$ for $\mathrm{Na}^{+}$and the absence of monovalent ions, see Table 2 ). In presence of $\mathrm{K}^{+}$both sequences display very high stabilities that cannot be melted completely, see Figure 2.

In contrast to the telomeric sequences, the short-looped sequences GQP1 and GQP2 fold into parallel-oriented four-stranded structures even in case of the DNA sequences, see Figure 3. This behavior has been reported before and is dependent on the presence of three single-nucleotide loops connecting the G-tracks. ${ }^{17}$ Expectedly, the corresponding RNAs as well fold very stable parallel quadruplexes. The RNA quadruplexes are more stable than DNA quadruplexes at all conditions studied for both oligonucleotides $G Q P 1$ and GQP2. Expectedly, the sequence GQP2 being able to form a quadruplex composed of only two stacked G-tetrads displays significantly lower melting temperatures compared to GQP1 for both DNA and RNA sequences. All sequences show very pronounced differences in stability with respect to the buffer conditions. More specifically, $\mathrm{K}^{+}$is stabilizing the parallel topologies much more compared to $\mathrm{Na}^{+}$-containing solutions. For the more stable GQP1, the structures are not disrupted even at temperatures as high as $90^{\circ} \mathrm{C}$. In addition, in case of GQP2 (Fig. $3 \mathrm{C}$ and D) only $\mathrm{K}^{4}$ is able to induce quadruplex formation in case of the DNA sequence whereas the corresponding RNA sequence is folded but very unstable.

Table 2

Melting temperatures of DNA and RNA quadruplexes. Estimated standard deviations determined by repetition of example measurements are less than $1{ }^{\circ} \mathrm{C}$

\begin{tabular}{|c|c|c|c|}
\hline Name & Buffer $\left({ }^{\circ} \mathrm{C}\right)$ & $100 \mathrm{mM} \mathrm{NaCl}(\mathrm{CO})$ & $100 \mathrm{mM} \mathrm{KCl}^{\mathrm{a}}\left({ }^{\circ} \mathrm{C}\right)$ \\
\hline HT DNA & $<20$ & 47 & 65 \\
\hline HT RNA & $<20$ & $42^{\prime}$ & 73 \\
\hline OXYDNA & $40^{\mathrm{a}}$ & 65 & $\$ 90$ \\
\hline OXY RNA & 35 & 60 & $>90$ \\
\hline GQP1 DNA & $39^{\mathrm{a}}$ & 62 & $>90$ \\
\hline GOP1 RNA & 50 & 73 & $>90$ \\
\hline COP2 DNA & $<20$ & $<20$ & 58 \\
\hline GQP2 RNA & $<20$ & $31 \%$ & 63 \\
\hline TBA DNA & $<20$ & $<20$ & 52 \\
\hline TBA RNA & $<20$ & $<20$ & 54 \\
\hline SDQ DNA & $<20$ & $<20$ & 42 \\
\hline SDQ RNA & 35 & 42 & 57. \\
\hline
\end{tabular}

\footnotetext{
${ }^{a}$ Only a fraction of the quadruplex is folded, the value represents the melting temperature of this fraction.
}

In case of the thrombin aptamer sequence, we observed an antiparallel quadruplex structure for the DNA sequence, see Figure 4A. The $C D$ signature corresponds to the chair conformation as has been demonstrated frequently for the TBA sequence. ${ }^{39.40}$

The stability of the two-stack aptamer quadruplex is rather low with only potassium being able to induce a completely folded structure. In contrast, the corresponding RNA sequence folds into the all-parallel conformation. A similar result has been obtained before, demonstrating that the exchange of a single residue in the 15 mer deoxyoligonucleotide with the respective ribonucleotide is sufficient to observe the parallel fold. ${ }^{30}$ Interestingly, the RNA sequence is slightly more stable than the TBA DNA. The strong preference of $\mathrm{K}^{+}$over $\mathrm{Na}^{+}$is observed in case of TBA RNA as well. With the sequence we have utilized in a bacterial reporter assay in order to block the Shine-Dalgarno sequence (SDQ), a parallel conformation is observed in the DNA as well as the RNA oligonucleotide. For the DNA sequence this finding is somewhat unexpected since it seems that sequences comprising short loops are favouring the parallel form in DNAs. ${ }^{16,17}$ However, in the SDQ-DNA sequence a conventional quadruplex composed of only two stacked tetrads can form, connected by rather extended loops. Accordingly, the thermal stabilities correspond to a quadruplex of rather low stability since only $\mathrm{K}^{+}$is able to induce the formation of the four-stranded sequence with a melting temperature of $42^{\circ} \mathrm{C}$. The corresponding RNA sequence folds into a parallel structure as well, being much more stable than the DNA sequence in the presence of $\mathrm{K}^{+}$(with a melting temperature of $57^{\circ} \mathrm{C}$, see Table 2). The high preference for $\mathrm{K}^{+}$versus $\mathrm{Na}^{+}$is also seen with the RNA sequence since the presence of $100 \mathrm{mM} \mathrm{Na}^{+}$is only resulting in partly folded structures at $25^{\circ} \mathrm{C}$, see Figure $4 \mathrm{D}$. This finding is very interesting since it might be possible that more complex structures with a central quadruplex might form in $\mathrm{K}^{+}$solutions. It has been found that RNA quadruplexes are able to scaffold other higher-order nucleotide assemblies. ${ }^{21,41.42}$

\section{Discussion}

In general, tendencies known to be specific for DNA quadruplexes have been found for the investigated RNA sequences as well. For example, the stability of the respective quadruplexes is highly 
A
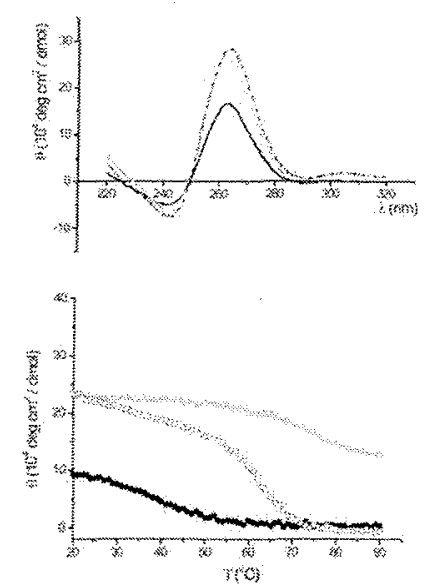

B
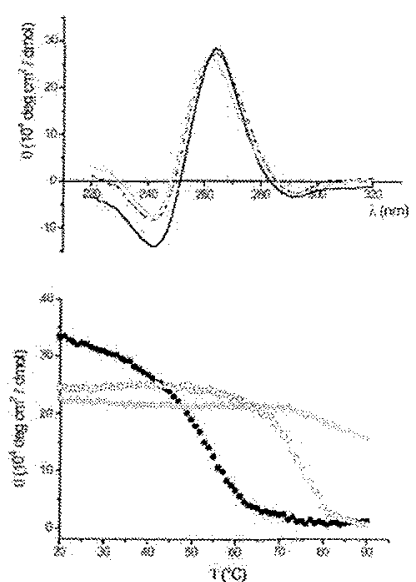

$\mathrm{C}$
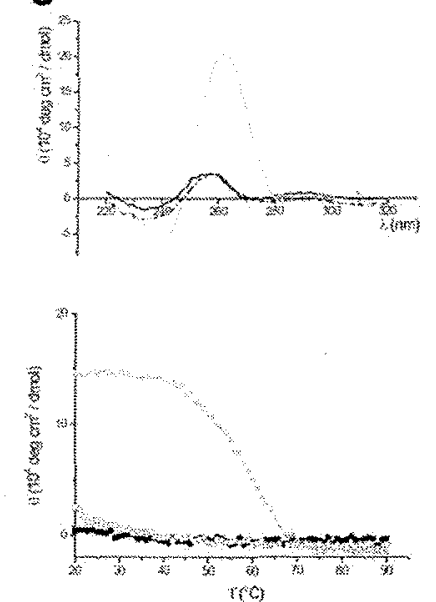

D
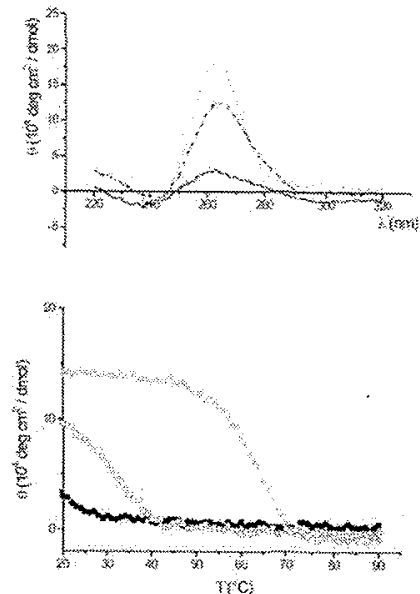

Figure 3. Short-loop sequences GQP1 and GQP2. (A) Short-loop DNA sequence composed of three G-tetrads (GOP1 DNA). (B) Short-loop RNA sequence composed of three Gtetrads (GQP1 RNA). (C) Short-loop DNA sequence composed of two G-tetrads (GQP2 DNA). (D) Short-loop RNA sequence composed of two G-tetrads (GQP2 RNA). Upper panels: $\mathrm{CD}$ spectra without monovalent ions (black line), containing $100 \mathrm{mM} \mathrm{NaCl}$ (dashed line) and $100 \mathrm{mM} \mathrm{KCl}$ (grey line). Lower panels: $\mathrm{CD}$ melting experiments carried out at the wavelength of the most pronounced peak in the upper $\mathrm{CD}$ spectra: without monovalent ions (black circles), containing $100 \mathrm{mM} \mathrm{NaCl}$ (open circles) and $100 \mathrm{mM} \mathrm{KCl}$ (grey circles).

A
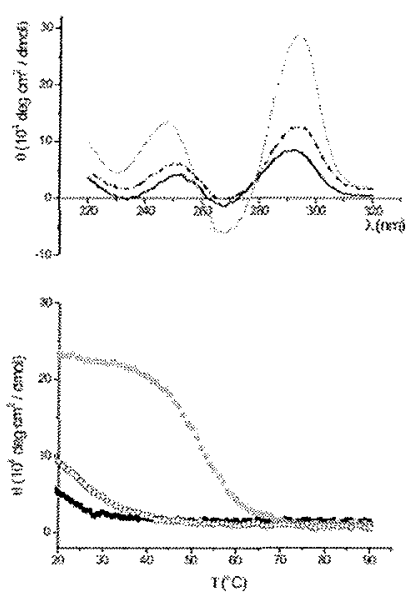

B
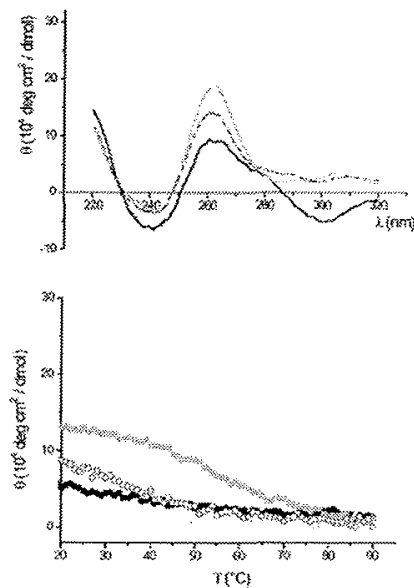

C
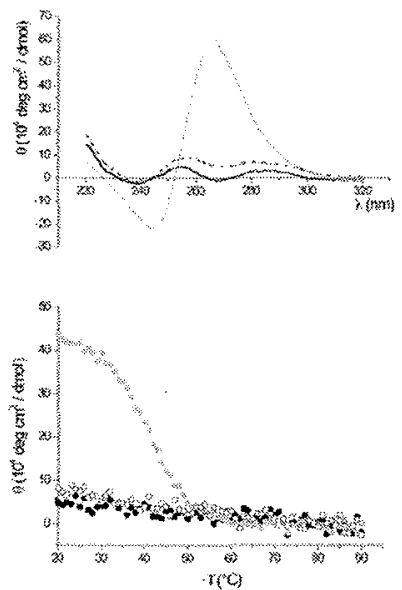

D
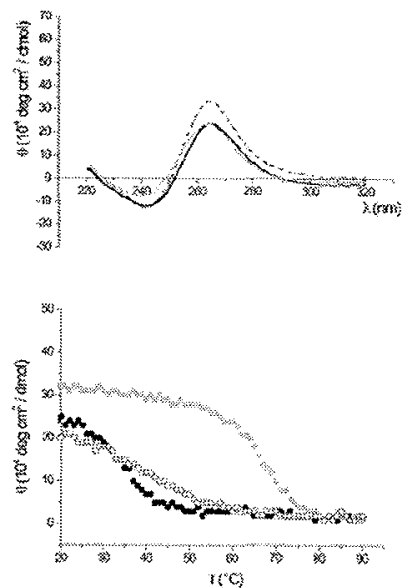

Figure 4. Thrombin aptamer and Shine-Dalgamo quadruplexes. (A) Thrombin-binding aptamer DNA sequence (TBA DNA). (B) Thrombin-binding aptamer RNA sequence (TBA RNA). (C) Shine-Dalgarno-containing DNA Quadruplex sequence (SDQ DNA). (D) Shine-Dalgarno-containing RNA Quadruplex sequence (SDQ RNA). Upper panels: CD spectra without monovalent ions (black line), containing $100 \mathrm{mM} \mathrm{NaCl}$ (dashed line) and $100 \mathrm{mM} \mathrm{KCl}$ (grey line). Lower panels: $\mathrm{CD}$ melting experiments carried out at the wavelength of the most pronounced peak in the upper CD spectra: without monovalent ions (black circles), containing $100 \mathrm{mM} \mathrm{NaCl}$ (open circles) and $100 \mathrm{mM} \mathrm{KCl}$ (grey circles).

dependent on the type of cation added: the stability increases in the series no cation $<\mathrm{Na}^{+}<\mathrm{K}^{+}$. Interestingly, in case of potassium which physiologically is also the most significant monovalent cation inside cells, almost all RNA quadruplexes (with the Oxytricha telomeric repeat as exception) form more stable four-stranded structures compared to their DNA counterparts. In general, in case of the short-looped sequences a higher stability of the RNA sequences is observed whereas with long loops (such as the Oxytricha four-nucleotide loops) the DNA quadruplexes display higher stabilities. A possible explanation for this observation is the preference of RNA quadruplexes to fold into parallel topologies. ${ }^{30,42}$ Although DNA sequences are able to adopt a variety of different quadruplex conformations, the all-parallel topology seems to be disadvantageous if other conformations are possible due to longer loop lengths. Only in case of single-nucleotide loops, the all-parallel form is dominant in DNA quadruplexes, forming a very stable structure. ${ }^{17}$

Computational studies have found thousands of putative quadruplex sequences in regions that are actively transcribed, potentially forming four-stranded RNA structures in vivo. An interesting distribution of putative quadruplexes was found both in pro- as well as in eukaryotes with respect to transcription start sites, untranslated regions, and open reading frames. ${ }^{25.43}$ We have recently provided strong evidence that RNA quadruplexes indeed form in vivo by placing $\mathrm{G}$-rich sequences into the ribosome binding site of a bacterial mRNA. ${ }^{18.19}$ Upon folding of the fourstranded sequence, ribosomal interaction with the mRNA is inhibited and the extent of the suppression correlates well with the 
thermodynamic stability of the quadruplex. In eukaryotes, quadruplexes have recently been found to suppress gene expression if present in 5'-UTRs. As the Balasubramanian and Kurreck groups have shown, RNA quadruplexes formed in the $N R A S^{22}$ and $Z l C-1^{23}$ mRNAs inhibit gene expression. Our group has shown that the suppressive effect of RNA quadruplexes in mRNAs is dependent on critical features such as loop length and number of $\mathrm{G}$-rich repeats in 5'-UTRs in mammalian cells. ${ }^{24}$ Given the unexpected stability of the SDQ RNA quadruplex with a melting temperature of almost $60^{\circ} \mathrm{C}$ in potassium-rich solutions, the formation of quadruplexes even from putative loci not immediately recognized as prerequisite quadruplex-forming sequences needs to be taken into account.

\section{Experimental section}

The respective DNA and RNA sequences (see Table 1) were ordered from MWG Biotech (Ebersberg. Germany) or synthesized using conventional oligonucleotide solid phase phosphoramidite chemistry (using TOM strategy for the RNA synthesis) and were purified by PAGE following standard deprotection procedures and analyzed by ESI-MS (HT-RNA: calcd 6904.2; meas. 6902.3; OXY-RNA: calcd 9134.4; meas. 9132.1; GQP1-RNA: calcd 4998.0; meas. 4996.8; GQP2-RNA: calcd 3617.2; meas. 3616.3; TBA-RNA: calcd 4880.9; meas. 4879.4; SDQ-RNA: calcd 8268.0; meas. 8266.4). For the circular dichroism studies, oligonucleotide samples were prepared at $5 \mu \mathrm{M}$ concentration in diethylpyrocarbonate-treated water buffered with $50 \mathrm{mM}$ Tris- $\mathrm{HCl}(\mathrm{pH} \mathrm{7.5)}$ and optional addition of $100 \mathrm{mM} \mathrm{KCl}$ or $\mathrm{NaCl}$. Oligonucleotides were annealed by heating to $95^{\circ} \mathrm{C}$ for $5 \mathrm{~min}$ followed by slow cooling to $20^{\circ} \mathrm{C}$ over $16 \mathrm{~h}$. CD spectra were recorded on a Jasco 715 spectrometer in cuvettes with a $1 \mathrm{~cm}$ path length, resolution of $0.5 \mathrm{~nm}$, band width of $1.0 \mathrm{~nm}$, and speed of $20 \mathrm{~nm} / \mathrm{min}$ at $20^{\circ} \mathrm{C}$. Each spectrum was accumulated five times and averaged. For thermal denaturation studies, the sample was heated from $20^{\circ} \mathrm{C}$ to $90^{\circ} \mathrm{C}$ with a heating rate of $1^{\circ} \mathrm{C} \mathrm{min}^{-1}$. The $\mathrm{CD}$ signal at $263 \mathrm{~nm}$ or $290 \mathrm{~nm}$ was recorded every $1^{\circ} \mathrm{C}$ and the melting temperature was obtained by determining the temperature at the half-maximum decrease of the signal.

\section{Acknowledgement}

J.S.H. gratefully acknowledges the VolkswagenStiftung for funding a Lichtenberg-Professorship, the Fonds der chemischen Industrie and the Zukunftskolleg for support.

\section{References and notes}

1. Patel, D. 1.: Phan, A. T.; Kuryavyi, V. Nucleic Acids Res. 2007, 35, 7429

2. Burge, S.: Parkinson, G. N.; Hazel, P.; Todd, A. K.; Neidle, S. Nucleic Acids Res. 2006, 34, 5402 .

3. Parkinson, G. N.; Lee, M. P.; Neidle, S. Nature 2002, 417, 876.

4. Wang, Y.; Patel, D. I. Structure 1993, 1, 263.

5. Phan. A. T.; Kuryavyi, V.: Luu, K. N.; Patel. D. J. Nucleic Acids Res. 2007, 35, 6517

6. Dai, J.; Carver, M.; Punchihewa, C.; Jones, R. A.; Yang. D. Nucleic Acids Res. 2007 $35,4927$.

7. Dai, J.; Carver, M.; Yang, D. Biochimie 2008, 90, 1172.

8. Rankin, S.: Reszka, A. P.: Huppert, J.; Zloh, M.: Parkinson, G. N.; Todd, A. K. Ladame, S.: Balasubramanian, S.: Neidle, S. J. Am. Chem. Soc. 2005, 127, 10584

9. Huppert. J. L.; Balasubramanian. S. Nucleic Acids Res. 2005, 33, 2908.

10. Todd, A. K.: Johnston, M.; Neidle, S. Nucleic Acids Res. 2005, 33, 2901

11. Phan, A. T.; Modi, Y. S.; Patel, D. J. J. Am. Chem. Soc, 2004, 126, 8710.

12. Dai, J.; Dexheimer, T. S.; Chen, D.; Carver, M.; Ambrus, A.: Jones, R. A.; Yang, D.J Am. Chem. Soc. 2006, 128, 1096.

13. Siddiqui-Jain, A.; Grand, C. L.; Bearss, D. J.; Hurley, L. H. Proc. Natl Acad. Sci U.S.A. 2002,99, 11593.

14. Rangan, A.; Fedoroff, O. Y.: Hurley, L. H. J. Biol. Chem. 2001. 276, 4640.

15. Phan, A. T.; Kuryavyi, V.: Gaw, H. Y.; Patel, D. J. Nat. Chem. Biol. 2005, 1, 167.

16. Bugaut, A.; Balasubramanian, S. Biochemistry 2008, 47, 689.

17. Hazel, P.; Huppert, J.; Balasubramanian, S.; Neidle, S. J. Am. Chem. Soc. 2004, $126,16405$.

18. Wieland, M: Hartig. J. S. Chem. Biol. 2007, 14, 757

19. M. Wieland, J. S. Hartig, Nature Protocols, in press.

20. Shafer, R. H.; Smirnov, I. Biopolymers 2000, 56, 209.

21. Pan, B.; Shi, K.; Sundaralingam, M. Proc. Natl. Acad. Sci. U.S.A. 2006, 103. 3130

22. Kumari. S.: Bugaut. A.: Huppert, J. L.; Balasubramanian, S. Nat. Chem. Biol. 2007 25,25

23. Arora, A.; Dutkiewicz, M.; Scaria, V.; Hariharan, M.; Maiti, S.; Kurreck, J. RNA 2008, 14,1290

24. K. Halder, M. Wieland, J. S. Hartig, Nucleic Acids Res. in press.

25. Huppert, J. L; Bugaut, A.; Kumari, S.; Balasubramanian, S. Nucleic Acids Res $2008,36,6260$.

26. Azzalin, C. M.; Reichenbach, P.; Khoriauli, L.; Giulotto, E; Lingner, J. Science 2007. 318. 798 .

27. Schoeftner, S.; Blasco, M. A. Nat. Cell Biol. 2008, 10, 228.

28. Xu, Y.: Kaminaga, K.: Komiyama, M. J. Am. Chem. Soc. 2008, 130, 11179

29. Martadinata, H.; Phan, A. T. J. Am. Chem. Soc. 2009, 131, 2570.

30. Tang, C. F.; Shafer, R. H. J. Am. Chem. Soc. 2006, 128, 5966

31. Wieland, M.; Hartig, J. S. Angew. Chem., Int. Ed. 2006, 45, 5875

32. Henn, A.; Joachimi, A.; Goncalves, D. P.; Monchaud, D.; Teulade Fichou, M. P. Sanders, J. K.: Hartig, J. S. Chembiochem 2008, 9, 2722.

33. Lim, K. W.; Amrane, S.: Bouaziz, S.: Xu, W.: Mu, Y.; Patel, D. J.: Luu, K. N.; Phan, A. T. J. Am. Chem. Soc. 2009, 131, 4301.

34. Wang. Y.; Patel, D. J. J. Mol. Biol. 1995, 251, 76

35. Haider, S.; Parkinson, G. N.; Neidle, S. J. Mol. Biol. 2002, 320, 189.

36. Bock, L. C.: Griffin, L. C.; Latham. J. A.: Vermaas, E. H.: Toole, J. J. Nature 1992 355,564

37. Schultze, P.: Macaya, R. F.; Feigon, J. J. Mol. Biol. 1994, 235, 1532

38. Smith, F. W.: Schultze, P.: Feigon, J. Structure 1995, 3, 997.

39. Feigon, J.: Dieckmann, T.; Smith, F. W. Chem. Biol. 1996, 3, 611

40. Padmanabhan, K.; Padmanabhan, K. P.; Ferrara.J. D.; Sadler, J. E.; Tulinsky, A.J Biol. Chem. 1993, 268, 17651.

41. Pan, B.; Xiong, Y.; Shi, K.: Sundaralingam, M. Structure 2003, 11, 1423.

42. Liu, H.; Matsugami, A.; Katahira, M.; Uesugi, S. J. Mol. Biol. 2002, $322,955$.

43. Rawal, P.; Kummarasetti, V. B.; Ravindran, J.; Kumar, N.; Halder, K.; Sharma, R. Mukerji, M.; Das, S. K.: Chowdhury. S. Genome Res. 2006, 16, 644. 